\title{
Mycota (Ascomycota) of Syagrus coronata (Mart.) Becc., Raso da Catarina Ecological Station, Brazil: new records
}

\author{
Nadja Santos Vitória ${ }^{*} \bullet$, Nilo Gabriel Soares Fortesa®, Maiara Araújo Lima dos Santosb®, \\ Rebeca Leite Barbosa ${ }^{a}$ \\ a Programa de Pós-Graduação em Biodiversidade Vegetal, Universidade do Estado da Bahia, Paulo Afonso, 48600-000, Bahia, Brasil. \\ *nadjasv@hotmail.com \\ b Programa de Pós-Graduação em Biologia de Fungos, Universidade Federal de Pernambuco, Recife, 50740-570, Pernambuco, Brasil.
}

Received: January 7, 2020 / Accepted: April 14, 2020 / Published online: May 25, 2020

\begin{abstract}
The Raso da Catarina Ecological Station (ESEC) is a conservation unit of integral protection located in the Caatinga biome, Northeastern Brazil, Bahia, between the cities of Paulo Afonso, Rodelas, and Jeremoabo. Among the botanical species of great relevance in the ESEC region stands out the palm Syagrus coronata (Mart.) Becc. (licuri). Currently, there is little information about the mycota that colonizes this host. Thus, this study analyzes the taxonomy of fungi occurring in $S$. coronata at ESEC Raso da Catarina. For that purpose, we conducted eight excursions between May 2014 and January 2015, in which we randomly demarcated and georeferenced 25 S. coronata plants. For the survey of the Ascomycota, we collected leaves, bracts, inflorescences, fruits, stem pieces and litter. We morphologically identified twenty-six taxa. Following an extensive literature research, we present dichotomous keys for genera, distribution data, and a checklist of fungi species, of which four are lichenized and 22 non-lichenized (nine anamorph/asexual and 13 teleomorph/sexual). These species belong to 26 genera and 18 families. We report Syagrus coronata in this study as a new botanical host for 22 fungi species.
\end{abstract}

Keywords: Caatinga, fungi, palm tree, taxonomy.

\section{Micota (Ascomycota) de Syagrus coronata (Mart.) Becc., Estação Ecológica Raso da Catarina, Brasil: novos registros}

\begin{abstract}
Resumo
A Estação Ecológica Raso da Catarina (ESEC) é uma unidade de conservação de proteção integral localizada no Bioma Caatinga, Nordeste do Brasil, estado da Bahia entre os municípios de Paulo Afonso, Rodelas e Jeremoabo. Dentre as espécies botânicas de grande relevância na região da ESEC destaca-se a palmeira Syagrus coronata (Mart.) Becc. (licuri). Na atualidade, são escassas as informações sobre a micota que coloniza esse hospedeiro. Assim, esta pesquisa objetivou realizar um estudo taxonômico dos fungos encontrados em S. coronata na ESEC Raso da Catarina. Oito excursões foram realizadas no período de maio/2014 a janeiro/2015 e 25 indivíduos foram demarcados aleatoriamente e georreferenciados. Para o levantamento dos Ascomycota, foram coletados folhas, brácteas, inflorescências, frutos e pedaços de tronco da parte aérea e serrapilheira dos indivíduos. Vinte e seis táxons foram identificados morfologicamente. Chaves dicotômicas para gêneros, dados de distribuição e uma lista de espécies de fungos, sendo quatro liquenizados e 22 não liquenizados (nove anamorfos/assexuais e 13 teleomorfos/sexuais), são apresentados baseados em uma intensa pesquisa de registros de literatura. Essas espécies estão distribuídas em 26 gêneros e 18 famílias. Syagrus coronata é documentada, neste estudo, como novo hospedeiro botânico para 22 espécies de fungos estudadas.
\end{abstract}

Palavras-chave: Caatinga, fungos, palmeira, taxonomia.

\section{Introduction}

Caatinga is the predominant type of vegetation in the Brazilian Northeast (Sena 2011). It covers about $11 \%$ of the national territory $\left(844,453 \mathrm{Km}^{2}\right)$, present in the states Alagoas, Bahia, Ceará, Maranhão, Pernambuco, Paraíba, Rio Grande do Norte, Piauí, Sergipe and northern Minas Gerais (Ministry of the Environment [MMA] 2012). According to Velloso,
Sampaio \& Pareyn (2002), most of the Caatinga biome is characterized by a hot and semiarid climate, with little rainfall. The soils of the semiarid region have a complex spatial distribution, from shallow and rocky, to sandy and deep, presenting a diversity of environments provided by a mosaic of types of vegetation. Velloso et al. (2002) suggest that the Caatinga is subdivided into eight ecoregions, among them the Raso da Catarina ecoregion. 
Raso da Catarina is an ecoregion located in the center-east of the Caatinga biome, has a narrow and elongated shape, occupying an area of approximately $30,800 \mathrm{~km}^{2}$ (Paes \& Dias 2008). There are five conservation units present: Raso da Catarina Ecological Station (ESEC Raso da Catarina), Serra Negra Biological Reserve, Canudos State Park, Canudos Biological Station and RPPN Fazenda Flor de Lis (Velloso et al. 2002). ESEC Raso da Catarina is located in the state of Bahia, between the municipalities of Paulo Afonso, Rodelas and Jeremoabo, standing out for its biological importance due to the diversity of flora and fauna.

Syagrus coronata (Mart.) Becc. is a palm well adapted to the dry and arid regions in the Caatinga biome. It is regionally called 'licurizeiro', 'licuri', 'ouricuri', 'auricuri', 'alicuri', 'nicuri', 'dicori', 'uricuri', 'coqueiro dicori', and 'coqueiro cabeçudo' (Drumond, 2007). The species has great socioeconomic importance in naturally occurring areas, especially in semiarid regions, since practically all parts of the plant can be used (Drumond, 2007; Lopes, 2007; Ramalho, 2008).

Several studies in the last decades have addressed mycota associated with Arecaceae, showing high species richness and numerous new species especially anamorphic microfungi and non-lichenized teleomorphs, which, because they are inconspicuous, are poorly studied and practically unknown (Hyde \& Fröhlich, 2000; Vitoria et al., 2008, 2011ab, 2012ab, 2014, 2016ab; Santos et al., 2016; Santos \& Vitoria, 2017; Vitoria et al., 2019; Santos et al., 2019).

Hyde (1996a), Fröhlich \& Hyde (1999) reported how many species of fungi could occur in a single palm, suggesting the ratio palm: fungi of 1:26 and 1:33, respectively, which would directly imply the global number of diversity of fungi that is currently estimated at 12 million (Wu et al. 2019).

Endophytic, phytopathogenic, saprobic, mutualist, and symbiont fungi inhabit palm trees, in which species of the phylum Ascomycota predominate. This phylum corresponds to the largest group in the Fungi kingdom, containing approximately sixty-six thousand genera, as estimated in the Outline of Ascomycota: 2017 (Wijayawardene et al., 2018).
Brazil is a country rich in palm species, some of which are of great social and economic importance for the northeast region. An example is $S$. coronata, called "the semiarid green gold". Currently, the mycota of this host is little known.

Thus, this research carried out a taxonomic study of the fungi found in S. coronata at ESEC Raso da Catarina, Paulo Afonso, Bahia, Brazil, presenting dichotomous keys, geographic distribution and a list of morphologically characterized species.

\section{Materials and Methods}

\section{Study Area}

The research was carried out at the Raso da Catarina Ecological Station (ESEC), located in northeastern Bahia State, between parallels $9^{\circ} 33^{\prime} \mathrm{S}$ and $9^{\circ} 54^{\prime} \mathrm{S}$ and meridians $39^{\circ} 29^{\prime} \mathrm{W}$ and $38^{\circ} 44^{\prime} \mathrm{W}$, on the left bank of the Vaza Barris River, and on the right bank of the São Francisco River (Paes $\&$ Dias 2008). The study area is approximately $473 \mathrm{~km}$ away from Salvador city, capital of the state.

\section{Material collection}

We conducted eight excursions between May 2014 amd January 2015. During the collections, we randomly demarcated and georeferenced 25 S. coronata plants (in flowering and fruiting) in five areas of ESEC, with a minimum distance of $100 \mathrm{~m}$ between plants. For the survey of the Ascomycota, we collected leaves, bracts (spathe), inflorescences, fruits, and stem pieces from shoots and litter (Figure 1).

The botanical material was fractionated, with the aid of puncturating materials, in fragments of 10 to $20 \mathrm{~cm}$ in length and subsequently packed in $2 \mathrm{~kg} \mathrm{Kraft}$ paper bags, identified with the following data: location, date, substrate, geographical coordinate, collector and collection number.

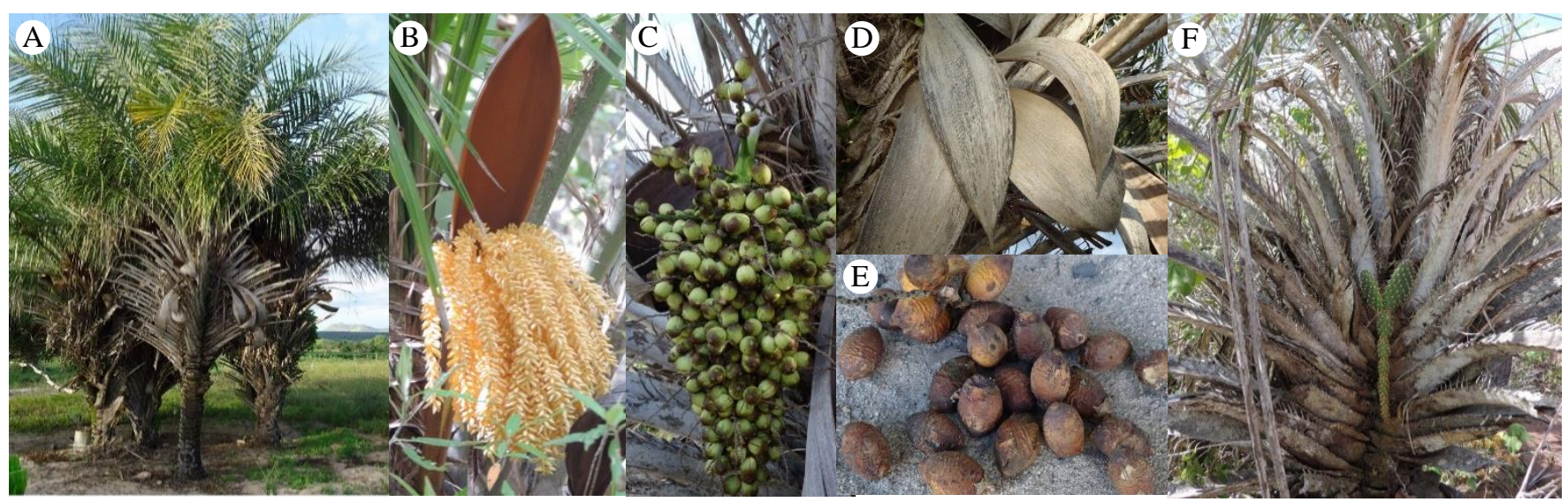

Figure 1. Material collected from Syagrus coronata to survey the Ascomycota. A: S. coronata in its natural environment; B: Inflorescence; C: Fruit bunch; D: Bracts; E: Ripe fruits; F: Petioles arranged in a crown. Photos: Fortes, N.G.S. and Santos, M.A.L. 


Morphological characterization, identification and
preservation

Wet chambers were mounted on plastic trays, properly cleaned with $70 \%$ alcohol and $2 \%$ sodium hypochlorite. The bottom and walls of the containers were covered with moistened paper towels. Every two days, the trays were opened to spray distilled water in order to maintain humidity. The plant material was stored for seven days, at room temperature and under natural light, to assess fungal growth. Subsequently, the topographic analysis of the material was performed in a stereomicroscope.

The fragments of the fungal structures were removed with the aid of a fine-tipped needle (type insulin) and mounted between slide and cover slip, using the following reagents: lactophenol with the addition of blue cotton dye to show hyaline structures; Melzer 5\% for diagnosis of the amyloid reaction at the apex of the asci and lichenic structures; $10 \%$ potassium hydroxide $(\mathrm{KOH})$ for dissolving protein material and water, as a means of assembly for visualizing the mucilaginous sheath, when present, around the structures. In addition, the preparation of the vertical sections of the ascomas and stems were carried out with the aid of razor blades and performed freehand to observe the reproductive structures.

We analyzed the samples at the Science Laboratory of the State University of Bahia - UNEB, Campus VIII, Paulo Afonso-BA. We identified fungi based on the morphology of the reproductive structures according to the relevant bibliography. Relevant (Arx \& Müller 1954; Müller \& Arx
1962; Ellis 1971, 1976; Luttrell 1973; Barnett \& Hunter 1998; Fröhlich \& Hyde 2000; Taylor et al. 2003; Cáceres 2007). After identification, we deposited the analyzed material in the URM Herbarium of the Federal University of Pernambuco (UFPE), in Recife city, and in the MICOLAB UNEB-VIII of the State University of Bahia (UNEB), in Paulo Afonso city.

We divided the fungi of this study into three major groups, namely: anamorphs, non-lichenized teleomorphs, and lichenized teleomorphs. We distributed the taxa in alphabetical order, presenting dichotomous keys for genera, distribution and material examined. Nomenclature and authors follow the online databases Species Fungorum (http://www.speciesfungorum.org/Names/Names.asp) and Index Fungorum (http://www.indexfungorum.org/ Names / Names.asp).

\section{Results and Discussion}

\section{Anamorphs}

Anamorphic or mitosporic fungi, that is, with asexual reproduction according to mycology references. This session shows fungi that produce conidia (asexual/mitotic spores) in solitary conidiophores, aggregated conidiophores (synnemata), and conidiophores inside conidiomata (pycnidia).

Identification key for the anamorphic genera of this study (Figure 2.A-U)

1. Conidia helicoid .2

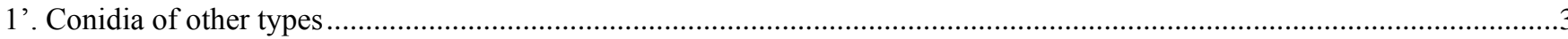

2. Conidia hyaline or shiny, with hygroscopic filaments .................................................................... Neohelicomyces

2'. Conidia brown or pale, nonhygroscopic filaments ............................................................................. Helicoma

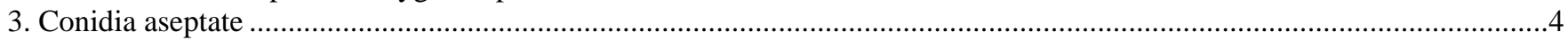

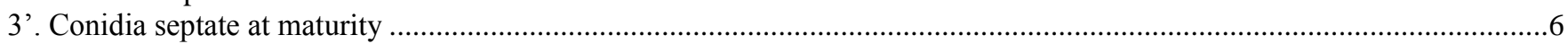

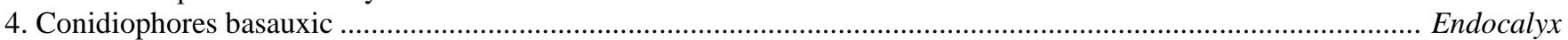

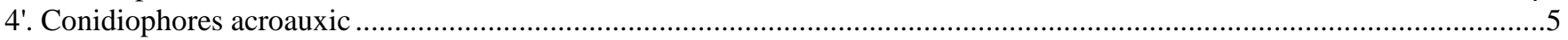

5. Conidia solitary, dry, and hyaline ................................................................................................. Phaeoisaria

5'. Conidia aggregated into sticky heads or dry chains brown to blackish .................................................. Stachybotrys

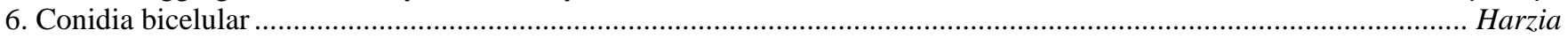

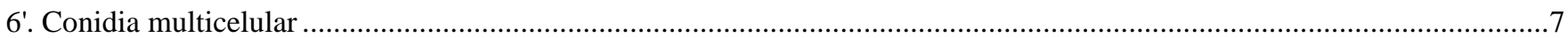

7. Conidia of two types: a spiny; b smooth, with irregularly lobed edges........................................................ Spegazzinia

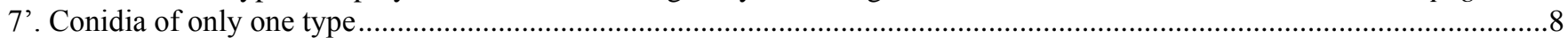

8. Conidia branched into chains, with constricted septa....................................................................................... Latorua

8'.Conidia solitary, with 4 columns of cells, ending at the apex with setous appendages ...................................... Tetraploa

\section{Endocalyx melanoxanthus (Berk. \& Broome) Petch}

Bibliography consulted: Ellis (1971), Vitoria et al. (2011b).

Distribution: Known distribution in Argentina, Australia, Brazil, China, Cuba, Philippines, Ghana, Hawaii, Hong Kong, Jamaica, Japan, Malaysia, Myanmar, Mexico, New Guinea, New Zealand, Pakistan, Sierra Leone, Seychelles, Singapore, Thailand, Taiwan, the United States, and Venezuela, colonizing hosts of the genera Acrocomia Mart., Archontophoenix H.Wendl. \& Drude, Arenga Labill., Borassus L., Chrysalidocarpus Becc., Cocos L., Coffea L., Dracaena Vand. ex L., Elaeis Jacq., Euterpe Mart., Licuala Wurmb,
Livistona R.Br., Nannorrhops H.Wendl., Oncosperma Blume, Phoenix, Ravenala Adans., Ripogonum J.R.Forst. \& G.Forst., Roystonea, Sabal, Satakentia H.E.Moore, Serenoa Hook.f., Shorea Roxb. ex C.F.Gaertn., Smilax L., Trachycarpus H.Wendl., Washingtonia H. Wendl., and Wodyetia Irvine (Farr \& Rossman, 2019). In Brazil, there are records of these specimens on the palms Acrocomia intumescens Drude and E. oleracea, for Pernambuco State, and E. dulis, for Bahia State (Vitoria et al., 2011b).

Material examined: Brazil. Bahia: Raso da Catarina Ecological Station (ESEC), Paulo Afonso city, on $S$. 
coronata bract, 01.15.2015, col. N.G.S. Fortes, 0949'82'’ S and $038^{\circ} 28^{\prime} 99^{\prime \prime} \mathrm{W}, 537 \mathrm{~m}$. (URM 91144 and MICOLAB UNEB-VIII 0006).

2. Harzia palmara (Cooke) D.W. Li \& N.P. Schultes, in Schultes, Murtishi \& Li

Bibliography consulted: Ellis (1976), Chen and Tzean (2009), Schultes et al. (2017).

Distribution: The genus is cosmopolitan, with diverse habitats: in association with plants, litter, seeds, soil, and manure. Known distribution in Canada, China, India, Ghana, Malaysia, Nepal, Nigeria, Papua New Guinea, Pakistan, Somalia, Taiwan, the United States, and Venezuela. In Brazil, there are records of this genus on Musa paradisiaca L. (Federal District), Sorghum bicolor (L.) Moench, and Vigna unguiculata (L.) Walp. (Pernambuco) (Farr \& Rossman, 2019; Mendes \& Urben, 2019).

Material examined: Brazil. Bahia: Raso da Catarina Ecological Station (ESEC), Paulo Afonso city, on $S$. coronata inflorescence, 06.10.2014, col. N.G.S. Fortes, 09³9'58' S and $38^{\circ} 28^{\prime} 46^{\prime \prime}$ 'W, $588 \mathrm{~m}$; on S. coronata leaflet, 01.15.2015, col. N.G.S. Fortes, $09^{\circ} 49^{\prime} 82^{\prime \prime}$ S and $038^{\circ} 28^{\prime} 99^{\prime \prime}$ W, $537 \mathrm{~m}$. (MICOLAB UNEB-VIII 0028).

\section{Helicoma microscopicum (Ellis) Linder}

Bibliography consulted: Zhao et al. (2007).

Distribution: Known distribution in China, colonizing decaying palm leaves, and in the USA (New Jersey), on Alnus serrulata (Aiton) Willd. (Farr \& Rossman, 2019).

Material examined: Brazil. Bahia: Raso da Catarina Ecological Station (ESEC), Paulo Afonso city, on S. coronata leaflet collected from the litter, 01.15.2015, col. N.G.S. Fortes, $09^{\circ} 49^{\prime} 82^{\prime \prime} \mathrm{S}$ and $38^{\circ} 28^{\prime} 99^{\prime \prime} \mathrm{W}, 537 \mathrm{~m}$. (MICOLAB UNEBVIII 0025).

\section{Latorua caligans (Bat. \& H.P. Upadhyay) Crous}

Bibliography consulted: Ellis (1971), Crous et al. (2015).

Distribution: Commonly occurring in the soil, with known distribution in Australia, India, Nigeria, and Brazil (in the states of Pernambuco and Sergipe). There are no records of $L$. caligans in other Brazilian states (Farr \& Rossman, 2019; Mendes \& Urben, 2019; Specieslink, 2019).

Material examined: Brazil. Bahia: Raso da Catarina Ecological Station (ESEC), Paulo Afonso city, on S. coronata leaflet, 01.15.2015, col. N.G.S. Fortes, $09^{\circ} 49^{\prime} 82^{\prime \prime}$ S and $038^{\circ} 28^{\prime} 99^{\prime \prime}$ W, 537 m. (URM 91146).

\section{Neohelicomyces pallidus (Cesati) Y.Z. Lu \& K.D. Hyde}

Bibliography consulted: Goos (1989), Zhao et al. (2007), Cruz et al. (2009), Lu et al. (2018).

Distribution: Known distribution in China (Guangdong, Hebei, Liaoning, Tibet), Czech Republic, Italy, Japan, and the USA (Massachusetts), on decaying wood. National databases do not contain information on the occurrence of N. pallidus (Lu et al., 2018; Specieslink, 2019; Farr \& Rossman, 2019; Mendes \& Urben, 2019). Therefore, this is the first record of the species in Brazil.

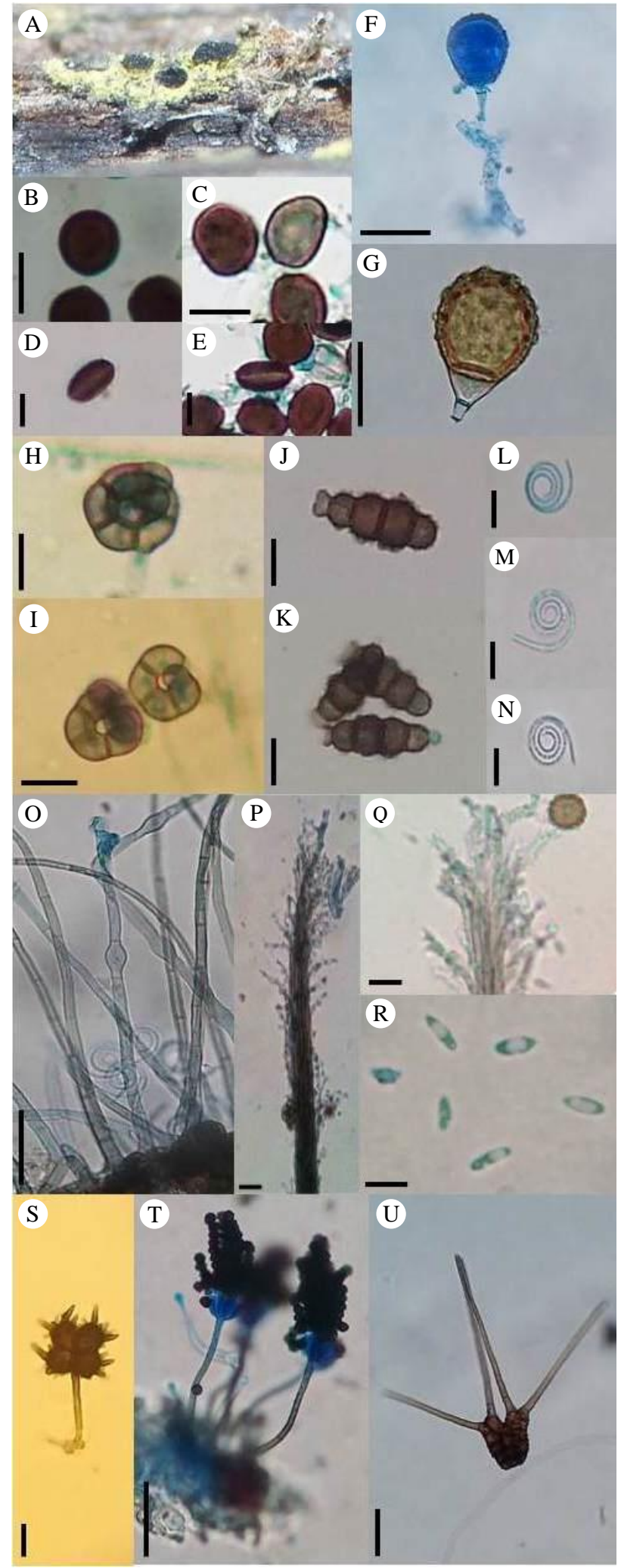

Figure 2. Anamorphic fungi. A-E: Endocalyx melanoxanthus; F-G: Harzia palmara; H-I: Helicoma microscopicum; J-K: Latorua caligans; L-O: Neohelicomyces pallidus; P-R: Phaeoisaria pseudoclematidis; S: Spegazzinia sp.; T: Stachybotrys echinatus; U: Tetraploa aristata. $(\mathrm{B}-\mathrm{C}=10 \mu \mathrm{m}, \mathrm{D}-\mathrm{E}=7,5$ $\mu \mathrm{m}, \mathrm{F}=30 \mu \mathrm{m}, \mathrm{G}=20 \mu \mathrm{m} \mathrm{H}-\mathrm{I}=7 \mu \mathrm{m}, \mathrm{J}-\mathrm{K}=10 \mu \mathrm{m}, \mathrm{L}-\mathrm{N}=$ $10 \mu \mathrm{m}, \mathrm{O}=20 \mu \mathrm{m}, \mathrm{P}=30 \mu \mathrm{m}, \mathrm{Q}=15 \mu \mathrm{m}, \mathrm{R}=7,5 \mu \mathrm{m}, \mathrm{S}=10$ $\mu \mathrm{m}, \mathrm{T}=30 \mu \mathrm{m}, \mathrm{U}=30 \mu \mathrm{m}$ ). 
Material examined: Brazil. Bahia: Raso da Catarina Ecological Station (ESEC), Paulo Afonso city, on S. coronata leaflet collected from the litter, 06.10.2014, col. N.G.S. Fortes, $09^{\circ} 40^{\prime} 10^{\prime \prime} \mathrm{S}$ and $38^{\circ} 27^{\prime} 87^{\prime \prime} \mathrm{W}, 531 \mathrm{~m}$. (MICOLAB UNEBVIII 0022).

\section{Phaeoisaria pseudoclematidis D.Q. Dai \& K.D. Hyde \\ Bibliography consulted: Ellis (1971), Liu et al. (2015).}

Distribution: The genus is cosmopolitan. Known distribution in Thailand only, with bamboo (Bambusa sp.) as host. This is the second record of this species in the world, with $S$. coronata as a new host for science (Liu et al., 2015; Farr \& Rossman, 2019).

Material examined: Brazil. Bahia: Raso da Catarina Ecological Station (ESEC), Paulo Afonso city, on $S$. coronata leaflet, 01.15.2015, col. N.G.S. Fortes, $09^{\circ} 49^{\prime} 82^{\prime \prime}$ S and $038^{\circ} 28^{\prime} 99^{\prime \prime}$ W, $537 \mathrm{~m}$. (URM 91148 and MICOLAB UNEB-VIII 0030).

\section{Spegazzinia sp.}

Bibliography consulted: Ellis (1971).

Distribution: The genus is cosmopolitan, with diverse habitats (Farr \& Rossman, 2019).

Material examined: Brazil. Bahia: Raso da Catarina Ecological Station (ESEC), Paulo Afonso city, on $S$. coronata bract, 01.15.2015, col. N.G.S. Fortes, $09^{\circ} 49^{\prime} 81^{\prime \prime}$ S and $038^{\circ} 28^{\prime} 98^{\prime \prime}$ W, 533 m. (MICOLAB UNEB-VIII 0021).

\section{Stachybotrys echinatus (Rivolta) G. Sm.}

Bibliography consulted: Wang et al. (2015).

Distribution: Known distribution in China, Honduras, Hong Kong, India, England, Japan, Malaysia, Kenya, Pakistan, Poland, Taiwan, and the USA (Farr \& Rossman, 2019). In Brazil, there are records of this species on decaying leaves of Miconia cabussu Hoehne (location not informed), Senna alata (L.) Roxb., and Ruellia incompta (Nees) Lindau (Bahia) (Mendes \& Urben, 2019; Barbosa et al., 2008).
Material examined: Brazil. Bahia: Raso da Catarina Ecological Station (ESEC), Paulo Afonso city, on $S$. coronata inflorescence, 06.10.2014, col. N.G.S. Fortes, $09^{\circ} 40^{\prime} 10^{\prime \prime} \mathrm{S}$ and $038^{\circ} 27^{\prime} 87^{\prime} \mathrm{W}, 531 \mathrm{~m}$. (MICOLAB UNEB-VIII 0031).

\section{Tetraploa aristata Berk. \& Broome}

Bibliography consulted: Ellis (1971), Tanaka et al. (2009).

Distribution: Known distribution in South Africa, Germany, Australia, Barbados, Bolivia, China, Cuba, Fiji, Ghana, the Virgin Islands, India, Italy, Hong Kong, Jamaica, Malaysia, Mexico, Myanmar, Papua New Guinea, Pakistan, Peru, Puerto Rico, Kenya, the United Kingdom, Dominican Republic, Sierra Leone, Thailand, Uganda, the USA, and Venezuela (Farr \& Rossman, 2019). In Brazil, there are records for the states of Bahia, Pará, Paraíba, Pernambuco, Rio Grande do Norte, Santa Catarina, and São Paulo (Specieslink, 2019).

Material examined: Brazil. Bahia: Raso da Catarina Ecological Station (ESEC), Paulo Afonso city, on $S$. coronata inflorescence, 01.15.2015, col. N.G.S. Fortes, $09^{\circ} 49^{\prime} 82^{\prime \prime} \mathrm{S}$ and $038^{\circ} 28^{\prime} 99^{\prime \prime} \mathrm{W}, 537 \mathrm{~m}$; on S. coronata leaflet collected from the litter, 01.15.2015, col. N.G.S. Fortes, $09^{\circ} 49^{\prime} 82^{\prime \prime} \mathrm{S}$ and $038^{\circ} 28^{\prime} 99^{\prime}$ ' W, $537 \mathrm{~m}$. (URM 91163 and MICOLAB UNEB-VIII 0033).

\section{Teleomorphs}

Fungi with sexual reproduction by meiotic spores formed inside the asci. The same as teleomorphic or meiosporic fungi, that is, with sexual reproduction. This session shows fungi that produce ascospores (sexual spores or meiospores) in perithecium-like, thyriothecium-like, and cleistotheciumlike ascomata.

\section{Teleomorphs Non-lichenized}

Fungi that do not associate with algae and/or cyanobacteria through lichenization.

Identification key for the non-lichenized teleomorphic genera of this study (Figura 3.A-Z)

1. Asci unitunicate or prototunicate .2

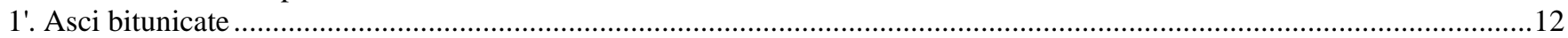

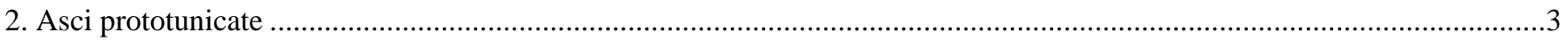

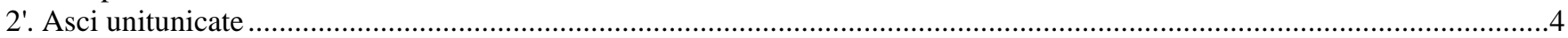

3. Cleistothecium surrounded by Hülle cells; reddishbrown ascospores............................................................. Emericella

3'. Cleistothecium pale yellow, shiny; hyaline ascospores ................................................................................... Eurotium

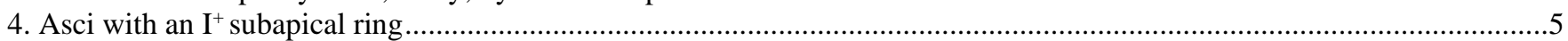

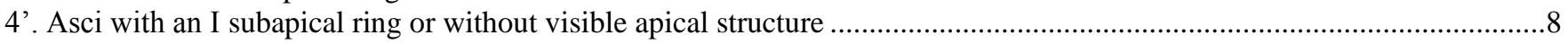

5. Ascomata lenticular; filiform ascospores, with 1 median septum ................................................................... Oxydothis

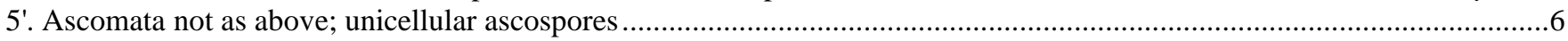

6. Ascospores hyaline, with a layered mucilaginous sheath; occasionally brown at maturity and with a germinative tube.

6'. Ascospores brown at maturity, with a nonlayered mucilaginous sheath

7. Ascospores with germinative tube, brown ascospores and occasionally with a dwarf cell.................. Fasciatispora

7'. Ascospores without germinative tube, with a pale median band

8. Ascomata stromatic

8 '. Ascomata of other types... 
9. Asci 8-spored; hyaline, ellipsoid ascospores surrounded by a mucilaginous sheath..... Camarotella

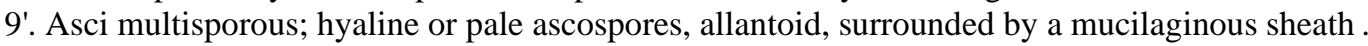
Diatrypella 10. Ascomata lenticular, semi-immersed under a blackish clypeus Linocarpon 10'. Ascomata not as above. . .11

11. Perithecium oval or globular, deeply immersed under a slightly raised or flattened clypeus Neolinocarpon

11 '. Perithecium almost or totally glabrous, with long neck; limoniform, apiculate, and smooth ascospores Melanospora

12. Thyriothecium linear, with epidermoid texture wall Aulographum

12 '. Perithecium Immersed; brown, bicellular ascospores Didymosphaeria

\section{Anthostomella palmaria B.S Lu \& K.D Hyde}

Bibliography consulted: Lu and Hyde (2000).

Distribution: Known distribution in the United States (Hawaii), Thailand, and Brazil (Bahia), colonizing the palms Cocos sp., Licuala longicalycata Furtado, and S. coronata (Lu \& Hyde, 2000; Farr \& Rossman, 2019; Specieslink, 2019).

Material examined: Brazil. Bahia: Raso da Catarina Ecological Station (ESEC), Paulo Afonso city, on the rachis of $S$. coronata leaves collected from the litter, 05.07.2014, col. N.G.S. Fortes, 09³9'44.9' ' S and 038²8'01.9'' W, 591 m. (URM 911149 and MICOLAB UNEB-VIII 0076).

\section{Aulographum sp.}

Bibliography consulted: The specimen is congeneric to Aulographum; we identified it based on the study by Sierra (2006).

Distribution: Aulographum species have a wide distribution, occurring as saprobes on plant remains (Farr \& Rossman, 2019).

Material examined: Brazil. Bahia: Raso da Catarina Ecological Station (ESEC), Paulo Afonso city, on S. coronata bract, 07.14.2014, col. N.G.S. Fortes, 09³9'58' 'S and 038 $28^{\circ}$ '04', W, 588 m. (URM 91152 and MICOLAB UNEB-VIII 0015).

\section{Camarotella torrendiella (Batista) Bezerra \& Vitoria}

Bibliography consulted: Vitoria et al. (2008).

Distribution: In Brazil, there are records for the states of Alagoas, Amapá, Amazonas, Bahia, Ceará, Espírito Santo, Pará, Paraíba, Pernambuco, Rio Grande do Norte, Rio de Janeiro, and Sergipe, on the palms Allagoptera brevicalyx M. Moraes, Attalea funifera Mart. ex Spreng., Bactris sp., B. pickelli Burret, B. ferruginea Burret, C. nucifera, Syagrus botryophora (Mart.) Mart., S. coronata, S. picrophylla Barb. Rodr., S. microphylla Burret, S. petraeae (Mart.) Becc., S. schizophylla (Mart.) Glassman, S. vagans (Bondar) A.D. Hawkes, and S. wedemannii Burret (Vitoria, 2010; Farr \& Rossman, 2019; Specieslink, 2019; Mendes and Urben, 2019).

Material examined: Brazil. Bahia: Raso da Catarina Ecological Station (ESEC), Paulo Afonso city, on S. coronata leaflet, 09.02.2014, col. N.G.S. Fortes, 09³9'39' 'S and 038 $27^{\circ}$ '44', W, 557 m. (URM 91165 and MICOLAB UNEB-VIII 0020).

\section{Capsulospora brunneispora K.D. Hyde}

Bibliography consulted: Hyde (1996b), Hyde \& Fröhlich (2000).

Distribution: Known distribution in Australia, Brazil, Brunei, Ecuador, Hong Kong, and Malaysia, colonizing the hosts
Archontophoenix alexandrae (F.Muell.) H.Wendl. \& Drude, Calamus australis Mart., Calamus conirostris Becc., $C$. nucifera, M. flexuosa, Phoenix hanceana Kunth (Arecaceae), and Pandanus tectorius Parkinson (Pandanaceae) (Farr \& Rossman, 2019; Specieslink, 2019).

Material examined: Brazil. Bahia: Raso da Catarina Ecological Station (ESEC), Paulo Afonso city, on $S$. coronata bract, 07.14.2014, col. N.G.S. Fortes, 09³9'58', S and $038^{\circ} 28^{\prime} 04^{\prime}$ ' W, $588 \mathrm{~m}$; on $S$. coronata bract collected from the litter, 07.14.2014, col. N.G.S. Fortes, 09³9'25', S and 038'29'28', W, $625 \mathrm{~m}$. (URM 91153 and MICOLAB UNEB-VIII 0049).

\section{Diatrypella caryotae R.K. Verma}

Bibliography consulted: Verma (1996).

Distribution: Known distribution in India, colonizing the palm Caryotae urens $\mathrm{L}$. This is the first record of this species in Brazil, with $S$. coronata as a new host for science (Verma, 1996).

Material examined: Brazil. Bahia: Raso da Catarina Ecological Station (ESEC), Paulo Afonso city, on $S$. coronata petiole, 05.07.2014, col. N.G.S. Fortes, $09^{\circ} 48^{\prime} 45^{\prime}$ ', $\mathrm{S}$ and $038^{\circ} 29^{\prime} 32^{\prime \prime} \mathrm{W}, 700 \mathrm{~m}$. (MICOLAB UNEB-VIII 0043).

\section{Didymosphaeria sp.}

Bibliography consulted: Lu and Hyde (2000).

Distribution: Genus whose species live in tropical regions (Farr \& Rossman, 2019).

Material examined: Brazil. Bahia: Raso da Catarina Ecological Station (ESEC), Paulo Afonso city, on $S$. coronata bract, 05.07.2014, col. N.G.S. Fortes, 0948'45', S and $38^{\circ} 29^{\prime} 32^{\prime}$ ' W, $700 \mathrm{~m}$. (MICOLAB UNEB-VIII 0065).

\section{Emericella sp.}

Bibliography consulted: Malloch and Cain (1972).

Distribution: The genus is cosmopolitan, with species in association with plants and in the soil (Farr \& Rossman, 2019).

Material examined: Brazil. Bahia: Raso da Catarina Ecological Station (ESEC), Paulo Afonso city, on $S$. coronata leaf rachis, 01.15.2015, col. N.G.S. Fortes, 09॰49'82', S and 038 $28^{\prime} 99^{\prime}$ ' W, $537 \mathrm{~m}$. (MICOLAB UNEB-VIII 0027).

\section{Eurotium sp.}

Bibliography consulted: Hubka et al. (2013). 
Distribution: The representatives of this group have a wide distribution in nature, being referred to as xerophiles or halophiles. Reports show that these individuals significantly impact the economy due to their capacity to degrade stored grains, cereals, textile products, herbarium materials, leather articles, among others (Hubka et al., 2013).

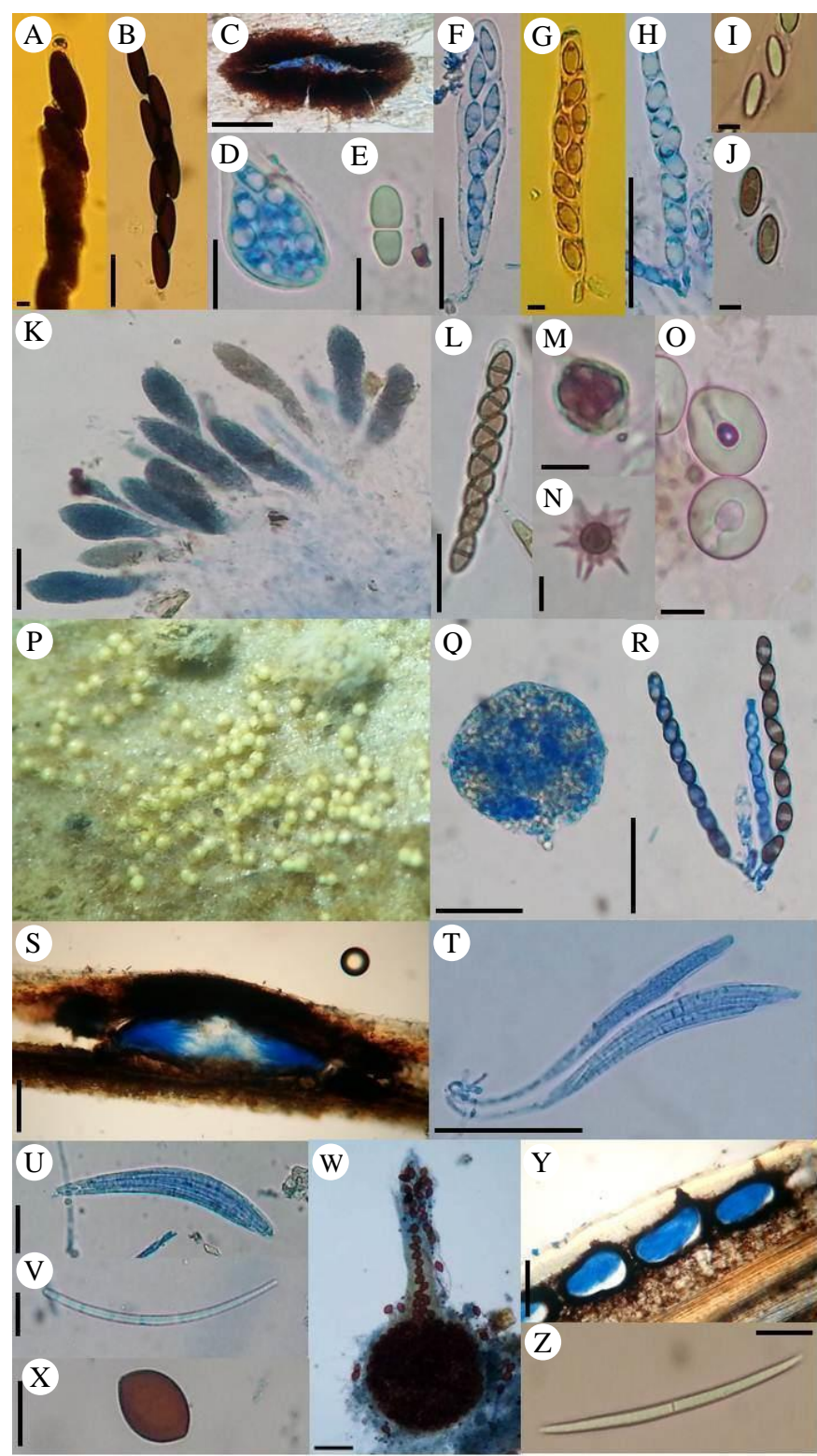

Figure 3. Non-lichenized Teleomorphic Fungi A-B: Anthostomella palmaria; C-E: Aulographum sp.; F: Camarotella torrendiella; G-J: Capsulospora brunneispora; K: Diatrypella caryotae; L: Didymosphaeria sp.; M-O: Emericella sp.; P-Q: Eurotium sp.; R: Fasciatispora petrakii; $\mathrm{S}-\mathrm{T}$ : Linocarpon sp.; U-V: Neolinocarpon attaleae; W-X: Melanospora zamiae; Y-Z: Oxydothis $\mathrm{sp} .(\mathrm{A}-\mathrm{B}=5 \mu \mathrm{m}, \mathrm{C}=75$ $\mu \mathrm{m}, \mathrm{D}=15 \mu \mathrm{m}, \mathrm{E}=10 \mu \mathrm{m}, \mathrm{F}=50 \mu \mathrm{m}, \mathrm{G}=4 \mu \mathrm{m}, \mathrm{H}=50 \mu \mathrm{m}, \mathrm{I}-$ $\mathrm{J}=7,5, \mathrm{~K}=30 \mu \mathrm{m}, \mathrm{L}=30 \mu \mathrm{m}, \mathrm{M}=10 \mu \mathrm{m}, \mathrm{N}=5 \mu \mathrm{m}, \mathrm{O}=20 \mu \mathrm{m}$, $\mathrm{Q}=40 \mu \mathrm{m}, \mathrm{R}=40 \mu \mathrm{m}, \mathrm{S}=180 \mu \mathrm{m}, \mathrm{T}=50 \mu \mathrm{m}, \mathrm{U}=20 \mu \mathrm{m}, \mathrm{V}=$ $30 \mu \mathrm{m}, \mathrm{W}=10 \mu \mathrm{m}, \mathrm{X}=75 \mu \mathrm{m}, \mathrm{Y}=50 \mu \mathrm{m}, \mathrm{Z}=25 \mu \mathrm{m})$.

Material examined: Brazil. Bahia: Raso da Catarina Ecological Station (ESEC), Paulo Afonso city, on S. coronata leaflet,
01.15.2015, col. N.G.S. Fortes, $09^{\circ} 49^{\prime} 81^{\prime \prime}$ S and $38^{\circ} 28^{\prime} 98^{\prime \prime}$ W, $533 \mathrm{~m}$; on S. coronata leaflet, 01.15.2015, col. N.G.S. Fortes, $09^{\circ} 49^{\prime} 82^{\prime \prime}$ S and $38^{\circ} 28^{\prime} 99^{\prime \prime} \mathrm{W}, 537 \mathrm{~m}$. (MICOLAB UNEB-VIII 0026).

\section{Fasciatispora petrakii (Mhaskar \& V.G. Rao) K.D. Hyde} Bibliography consulted: Hyde (1995).

Distribution: Known distribution in Australia, Brunei, China, Philippines, Florida (USA), India, Hong Kong, Java, Malaysia, Papua New Guinea, Singapore, and Thailand, colonizing hosts of the family Arecareae, belonging to the following genera: Archontophoenix, Butia (Becc.) Becc., Calamus L., Cocos, Cyrtostachys Blume, Daemonorops Blume ex Schult.f., Elaeis, Euterpe, Licuala, Livistona, Mauritia L.f., Metroxylon Rottb., Oncosperma, Phoenix, Sabal, Syagrus, Trachycarpus H.Wendl., and Wallichi (Farr \& Rossman, 2019). In Brazil, there are records of these specimens on Butia sp. (Federal District), E. oleifera (Bahia and Pernambuco), Elaeis guineensis, M. flexuosa, and S. botryophora (Pernambuco) (Mendes \& Urben, 2019; Specieslink, 2019).

Material examined: Brazil. Bahia: Raso da Catarina Ecological Station (ESEC), Paulo Afonso city, on $S$. coronata bract, 07.14 .2014 , col. N.G.S. Fortes, $09^{\circ} 39^{\prime} 58^{\prime \prime}$ S and $038^{\circ} 28^{\prime} 04^{\prime \prime} \mathrm{W}, 588 \mathrm{~m}$. (URM 91154 and MICOLAB UNEB-VIII 0002).

\section{Linocarpon sp.}

Bibliography consulted: Hyde (1997).

Distribution: Group whose species live mostly in tropical regions of Oceania (Farr \& Rossman, 2019). Currently, this genus comprises 43 valid epithets (Species Fungorum, 2019).

Material examined: Brazil. Bahia: Raso da Catarina Ecological Station (ESEC), Paulo Afonso city, on $S$. coronata petiole collected from the litter, 07.14.2014, col. N.G.S. Fortes, $09^{\circ} 47^{\prime} 18^{\prime \prime} \mathrm{S}$ and $038^{\circ} 29^{\prime} 31^{\prime \prime} \mathrm{W}, 685 \mathrm{~m}$. (MICOLAB UNEB-VIII 0010).

\section{Melanospora zamiae Corda}

Bibliography consulted: Cannon and Hawksworth (1982).

Distribution: This fungus is very widespread in the tropics and in warm temperate regions, frequently occurring on rotting vegetation or in association with animal waste. It has known distribution in Germany, Egypt, the USA (California, Florida, Illinois, and New York), Ghana, India, England, Israel, Libya, Tanzania, and Venezuela (Farr \& Rossman, 2019). In Brazil, there are records of these specimens on goat droppings, in Recife city, Pernambuco State (Specieslink, 2019).

Material examined: Brazil. Bahia: Raso da Catarina Ecological Station (ESEC), Paulo Afonso city, on $S$. coronata leaflet, 01.15 .2015 , col. N.G.S. Fortes, $09^{\circ} 49^{\prime} 82^{\prime \prime}$ $\mathrm{S}$ and $038^{\circ} 28^{\prime} 99^{\prime \prime} \mathrm{W}, 537 \mathrm{~m}$. (MICOLAB UNEB-VIII 0041). 


\section{Neolinocarpon attaleae N.S. Vitoria \& J.L. Bezerra}

Bibliography consulted: Vitoria et al. (2013).

Distribution: Vitoria et al. (2013) were the first authors to describe and illustrate this species, which colonized the palm A. funifera in Bahia State.

Material examined: Brazil. Bahia: Raso da Catarina Ecological Station (ESEC), Paulo Afonso city, on $S$. coronata petiole, 09.02.2014, col. N.G.S. Fortes, $09^{\circ} 39^{\prime} 39^{\prime \prime}$ S and $038^{\circ} 27^{\prime} 44^{\prime \prime}$ $\mathrm{W}, 557 \mathrm{~m}$.

\section{Oxydothis sp.}

Bibliography consulted: Hyde and Frohlich (2000).

Distribution: Its representatives occur in nature as saprobes, endophytes, or parasites, on leaves or petioles, usually causing deep and dark lesions. The literature refers to members of the families Arecaceae, Poaceae, Liliaceae, and Pandanaceae as their hosts. Currently, this genus comprises 79 valid epithets (Species Fungorum, 2019).

Material examined: Brazil. Bahia: Raso da Catarina Ecological Station (ESEC), Paulo Afonso city, on $S$. coronata petiole, 05.07.2014, col. N.G.S. Fortes, 0948'45', $\mathrm{S}$ and $038^{\circ} 29^{\prime} 32^{\prime \prime} \mathrm{W}, 700 \mathrm{~m}$; on $S$. coronata petiole collected from the litter, 10.21.2014, col. N.G.S. Fortes, $09^{\circ} 38^{\prime} 16^{\prime \prime} \mathrm{S}$ and $038^{\circ} 29^{\prime} 26^{\prime \prime} \mathrm{W}, 595 \mathrm{~m}$. (URM 91158 and MICOLAB UNEB-VIII 0007).

\section{Teleomorphs Lichenized}

Fungi that associate with algae and/or cyanobacteria through lichenization.

Identification key for the lichenized teleomorphic genera of this study (Figura 4.A-J)

1. Thallus crustose, grayishwhite

1'.Thallus crustose, yellowish-white; verruciform, solitary or gregarious apothecium, with the same color as the thallus; asci twospore, bitunicate ..... Pertusaria

2. Ascospores ellipsoid to fusiform, macrocephalic with transverse septa Arthonia

$2^{\prime}$. Ascospores muriform with transverse and longitudinal septa

3. Ascospores hyaline to pale yellow, smooth with lumina Cryptothecia

3'. Ascospores hyaline, smooth with mucilaginous sheath.... Polymeridium

\section{Coniocarpon cinnabarinum DC.}

Bibliography consulted: Cáceres (2007).

Distribution: In Brazil, there are records for the states of Alagoas, Pernambuco, Rio Grande do Sul, Rondônia, São Paulo, and Sergipe (Specieslink, 2019).

Material examined: Brazil. Bahia: Raso da Catarina Ecological Station (ESEC), Paulo Afonso city, on S. coronata petiole collected from the litter, 10.21.2014, col. N.G.S. Fortes, $09^{\circ} 38^{\prime} 16^{\prime \prime} \mathrm{S}$ and $038^{\circ} 29^{\prime} 26^{\prime \prime} \mathrm{W}, 595 \mathrm{~m}$; on $S$. coronata petiole, 10.21.2014, col. N.G.S. Fortes, $09^{\circ} 39^{\prime} 02^{\prime \prime}$ S $\mathrm{S}$ and $038^{\circ} 28^{\prime} 52^{\prime \prime} \mathrm{W}, 602 \mathrm{~m}$. (MICOLAB UNEB-VIII 0067).

\section{Cryptothecia sp.}

Bibliography consulted: Cáceres (2007).

Distribution: In Brazil, there are records of this genus in the states of Amapá, Amazonas, Federal District, Goiás, Mato Grosso do Sul, Minas Gerais, Pará, Paraná, Pernambuco, Piauí, Rio de Janeiro, Rio Grande do Sul, Rondônia, Santa Catarina, São Paulo, and Sergipe (Specieslink, 2019).

Material examined: Brazil. Bahia: Raso da Catarina Ecological Station (ESEC), Paulo Afonso city, on $S$. coronata petiole, 10.21.2014, col. N.G.S. Fortes, $09^{\circ} 38^{\prime} 02^{\prime \prime}$ S and $038^{\circ} 29^{\prime} 26^{\prime \prime}$ W, 599 m. (MICOLAB UNEB-VIII 0071).

\section{Pertusaria carneola (Eschw.) Müll. Arg.}

Bibliography consulted: Cáceres (2007).

Distribution: In Brazil, there are records for the states of Pernambuco, Piauí, Rio Grande do Sul, and Sergipe (Specieslink, 2019)

Material examined: Brazil. Bahia: Raso da Catarina Ecological
Station (ESEC), Paulo Afonso city, on S. coronata stem collected from the litter, 09.02.2014, col. N.G.S. Fortes, $09^{\circ} 37^{\prime} 39^{\prime \prime}$ S and $038^{\circ} 29^{\prime} 27^{\prime \prime}$ W, $596 \mathrm{~m}$; on S. coronata petiole, 05.07.2014, col. N.G.S. Fortes, $09^{\circ} 40^{\prime} 02^{\prime \prime} \mathrm{S}$ and $038^{\circ} 27^{\prime} 56^{\prime \prime} \mathrm{W}, 567 \mathrm{~m}$. (MICOLAB UNEB-VIII 0140).

\section{Polymeridium sp.}

Bibliography consulted: Cáceres (2007).

Distribution: In Brazil, there are records of this genus in the states of Bahia, Ceará, Espírito Santo, Minas Gerais, Pernambuco, Piauí, Rio Grande do Sul, Rondônia, and Sergipe (Specieslink, 2019).

Material examined: Brazil. Bahia: Raso da Catarina Ecological Station (ESEC), Paulo Afonso city, on $S$. coronata petiole, 10.21.2014, col. N.G.S. Fortes, $09^{\circ} 39^{\prime} 02^{\prime \prime}$ $\mathrm{S}$ and $038^{\circ} 28^{\prime} 52^{\prime \prime} \mathrm{W}, 602 \mathrm{~m}$; on $S$. coronata petiole collected from the litter, 10.21.2014, col. N.G.S. Fortes, $09^{\circ} 38^{\prime} 16^{\prime \prime} \mathrm{S}$ and $038^{\circ} 29^{\prime} 26^{\prime \prime}$ W, $595 \mathrm{~m}$; on S. coronata petiole collected from the litter, 07.14.2014, col. N.G.S. Fortes, 09 $40^{\prime} 06^{\prime \prime} \mathrm{S}$ and $038^{\circ} 27^{\prime} 47^{\prime \prime} \mathrm{W}, 536 \mathrm{~m}$. (MICOLAB UNEB-VIII 0069).

$S$. coronata occurs naturally in the Brazilian semiarid region and, although it is not an endangered species, its disordered and intensive use makes this host vulnerable (Silva et al., 2006).

The knowledge about the mycota of the palm S. coronata in the Caatinga biome is limited, being restricted to publications of a taxonomic nature of Cruz et al. (2009), that described and illustrated three species of Ascomycota: Piricauda cochinensis (Subram.) M.B. Ellis, Helicosporium virescens (Pers.) Sivan. and Repetophragma filiferum (Piroz.) R.F. Castañeda, Gusmão and Heredia, found in the 
municipalities of Senhor do Bonfim and Campo Formoso (Bahia); Santos et al. (2016) with 12 cataloged species, collected in Paulo Afonso, Juá-Bahia; Vitoria et al. (2016) with Checklist of 25 taxa from expeditions carried out at ESEC Raso da Catarina and Paulo Afonso in the Juá and Bogó-Bahia villages; Santos and Vitoria (2017) with seven descriptions of fungi found in Água Branca-Alagoas; Santos et al. (2019a) with a new record for the American continent collected in Paulo Afonso, Juá-Bahia and Santos et al. (2019b) presenting 20 lichenized and non-lichenized Ascomycota species collected in Paulo Afonso (Juá and Bogó villages) and Nova Glória (Brejo do Burgo and Serrota villages).

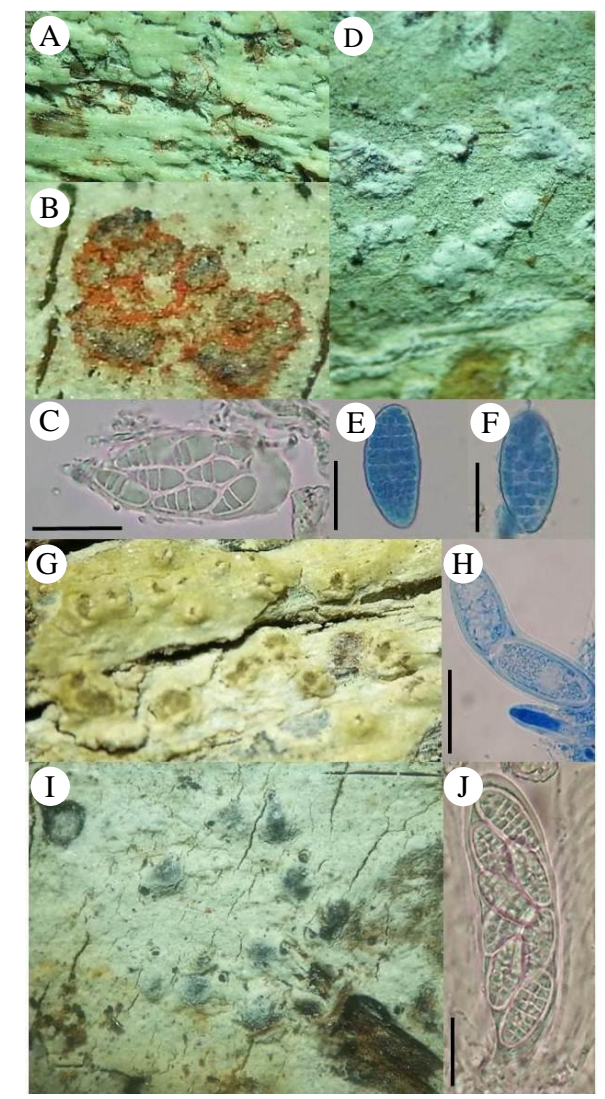

Figure 4. Lichenized Teleomorphic Fungi. A-C: Coniocarpon cinnabarinum; D-F: Cryptothecia sp.; G$\mathrm{H}$ : Pertusaria carneola; I-J: Polymeridium sp. $(\mathrm{C}=30$ $\mu \mathrm{m}, \mathrm{E}-\mathrm{F}=20 \mu \mathrm{m}, \mathrm{H}=60 \mu \mathrm{m}, \mathrm{J}=20 \mu \mathrm{m})$.

Currently, according to publications reporting studies on the $S$. coronata mycota, the occurrence record is less than 100 identified species. Thus, the new records presented in this research expand not only the information about $S$. coronata mycota collected at ESEC Raso da Catarina, but also enriches Brazilian mycology.

The number of fungal species on Arecaceae is usually large. Hyde (1996a) suggested for this family the palm:fungi ratio of 1:26, higher than the ratio of 1:6, generally accepted for other plants. Fröhlich and Hyde (1999) estimated 1:33 the palm: fungus ratio for the tropics. According to Pinruan et al. (2007), the morphology of palms may influence fungal colonization. In the present study, anamorphic (asexual) fungi occurred mostly on leaflets, while teleomorphic (sexual) fungi occurred on the petiole and leaf rachis. For Pinruan et al. (2007), palm parts exposed to different microhabitat conditions and substrate nutrient availability show differences in fungal populations. Palm stems and petioles provide a substrate rich in cellulose and lignin, and only fungi with the necessary enzymes can use it. Palm leaves favor anamorphic (asexual) species that colonize quickly, and dry after nutrient depletion.

Considering the information from publications and online databases such as SpeciesLink (2019), Embrapa Fungi Reported in Plants in Brazil (Mendes \& Urben, 2019), and Fungal databases - Fungus-Host Distributions (Farr \& Rossman, 2019), the results obtained in this study considerably increase the knowledge about the richness of fungal species sheltered by $S$. coronata, because this study reports $S$. coronata as a new substrate for 22 identified Ascomycota species.

\section{Conclusion}

The information we provided about the Ascomycota on $S$. coronata expands the knowledge about the taxonomy, geographic distribution, and ecology of the mycota in the Caatinga biome. In addition, it highlights the importance of this host for the native fungal community, suggesting the continuation of research. Future studies must focus on biological diversity, registration of new species, and the existing relationships between these organisms and the plant, with a view to developing biodiversity conservation strategies.

\section{Acknowledgments}

The authors thank the State University of Bahia (UNEB) for providing the facilities and laboratories to conduct this research; FAPESB (Foundation for the Support of Research in Bahia State) and CAPES (Coordination for the Improvement of Higher Education Personnel) for granting the scholarships; and ICMBio (Chico Mendes Institute for Biodiversity Conservation) for their support during collections at the Raso da Catarina Ecological Station (ESEC).

\section{References}

Arx, J. A. Von. \& Müller, E. (1954). Die Gattugen der Amerosporen Pyrenomyceten. Beitrage zur Kryptogamen flora der Schweiz, 11(1), 1434.

Barbosa, F. R., Gusmão, L. F. P. \& Barbosa, F. F. (2008). Fungos anamórficos (Hyphomycetes) no Semiárido do Estado da Bahia, Brasil. Acta Botânica Brasílica 22, 29-36. doi:10.1590/S010233062008000100004

Barnett, H. L. \& Hunter, B. B. (1998). Illustrated genera of Fungi Imperfecti. Minnesota, APS Press.

Cáceres, M. E. S. (2007). Corticolous crustose and microfoliose lichens of northeastern Brazil. IHW-Verlag, Eching, Alemanha. Libri Botanici 22: 1-168.

Cannon, P. F. \& Hawksworth, D. L. (1982). A re-evaluation of Melanospora Corda and similar Pyrenomycetes, with a revision of the British species. Botanical Journal of the Linnean Societ, 84: 115-160. doi:10.1111/j.1095-8339.1982.tb00363.x

Chen, J. L. \& Tzean, S. S. (2009). Hyphomycetes from Taiwan: Chaetendophragmia and Allied Species. Taiwania, 54(2): 152-158. doi: 10.6165/tai.2009.54(2).152

Crous, P. W., Carris, L. M., Giraldo, A., Groenewald, J. Z., Hawksworth, D. L., Hemández-Restrepo, M., ... \& Wood, A. R. (2015). The Genera 
of Fungi - fixing the application of the type species of generic names G2: Allantophomopsis, Latorua, Macrodiplodiopsis, Macrohilum, Milospium, Protostegia, Pyricularia, Robillarda, Rotula, Septoriella, Torula, and Wojnowicia. IMA Fungus. 6(1), 163-198. doi:10.5598/imafungus.2015.06.01.11

Cruz, A. C. R., Izabel, T. S., Leão-Ferreira, S. M. \& Gusmao, L. F. P. (2009). Conidial fungi from the semiarid Caatinga biome of Brazil. New species and new records of Helicosporium. Mycotaxon. 110, 53-64. doi: $10.5248 / 110.53$

Drumond, M. A. (2007). Licuri Syagrus coronata (Mart.) Becc. Petrolina: Embrapa Semiárido.

Ellis, M. B. (1971). Dematiaceous Hyphomycetes. Commonwealth Mycological Institute, Kew, U.K.

Ellis, M. B. (1976). More Dematiaceous Hyphomycetes. Kew, Commonwealth Mycological Institute.

Farr, D. F. \& Rossman, A. Y. (2019). Fungal Databases, U.S. National Fungus Collections, ARS, USDA.

Fröhlich, J. \& Hyde, K. D. (1999). Biodiversity of palm fungi in the tropics: are global fungal diversity estimates realistic? Biodiversity and Conservation, 8, 977-1004.

Fröhlich, J. \& Hyde, K. D. (2000). Palm Microfungi. Fungal Diversity Research Series 3.

Goos, R. D. (1989). On the anamorph genera Helicosporium and Drepanospora. Mycologia, 81, 356-374. doi: $10.1080 / 00275514.1989 .12025759$

Hubka, V., Kubatova, A. \& Peterson S.W. (2013). Taxonomic revision of Eurotium and transfer of species to Aspergillus. Mycologia, 105(4), 912937. doi:10.3852/12-151

Hyde, K. D. \& Fröhlich, J. (2000). Ascomycetes associated with palms. In: Bhat, D. J., Raghukumar, S. (eds.) Ecology of fungi. Goa, India, Goa University, pp. 109-114.

Hyde, K. D. (1995). Fungi from palms. XVII. The genus Fasciatispora, with notes on Amphisphaerella. Nova Hedwigia, 61(1-2): p. 249-268.

Hyde, K. D. (1996a). Measuring biodiversity: Diversity of microfungi in north Queensland. In: Bolye,T.J.B., Boontawee, B. (eds.) Measuring and monitoring Biodiversity in Tropical and Temperate Forest. Cifor: Indonesia, pp. 271-286.

Hyde, K. D. (1996b). Fungi from palms. XXVII. Capsulospora gen. nov., with three new species. Sydowia, 48(1), 111-121.

Hyde, K. D. (1997). Additions to the genus Linocarpon (Ascomycetes: Hyponectriaceae). Botanical Journal of the Linnean Society, 123(2), 109131. doi:10.1111/j.1095-8339.1997.tb01407.x

Liu, J. K., Hyde, K. D. Jones, E. B. G., Ariyawansa, H. A., Bhat, D. J., Boonmee, S... \& Camporesi. E. (2015). Fungal diversity notes 1-110: taxonomic and phylogenetic contributions to fungal species. Fungal Diversity. 72, 1-197. doi:10.1007/s13225-015-0324-y

Lopes, V. S. (2007). Morfologia e Fenologia Reprodutiva do Ariri (Syagrus vagans Bondar) Hawkes) Arecaceae numa área de caatinga do Município de Senhor do Bonfim-BA. Dissertação (Mestrado em Agronomia). Universidade Federal da Paraíba.

Lu, B. S. \& Hyde, K. D. (2000). Fungal Diversity Research Series 4. Hong Kong, Fungal Diversity Press.

Lu, Y. Z, Liu, J. K., Hyde, K. D., Jeewon, R., Kang, J. C., Fan,... \& Eungwanichayapant, P. D.(2018). A taxonomic reassessment of Tubeufiales based on multi-locus phylogeny and morphology. Fungal Diversity, 92, 131-344. doi:10.1007/s13225-018-0411-y

Luttrell, E. S. (1973). Loculoascomycetes. In: Ainsworth, G.C., Sparrow, F. K., Sussman, A.S. (eds.) The Fungi, an Advanced Treatise v.4 A. London, Academic Press, p. 135-219.

Malloch, D. \& Cain, R. F. (1972). New species and combinations of cleistothecial ascomycetes. Can J Bot 50:61-72. doi:10.1139/b72-011

Mendes, M. A. S. \& Urben, A. F. (2019). Fungos relatados em plantas no Brasil, Laboratório de Quarentena Vegetal. Brasília, DF: Embrapa Recursos Genéticos e Biotecnologia.

Ministério do Meio Ambiente. (2012). Caatinga. Brasília, Ministério do Meio Ambiente.

Müller, E. \& Arx, J. A. Von. (1962). Die Gattungen der Didymosporen Pyrenomyceten. Beitrage zur Kryptogamenflora der Schweiz v. 11,. p. 1922.

Paes, M. L. N. \& Dias, I. F. (2008). Plano de manejo: Estação Ecológica Raso da Catarina. Brasília: Ibama.

Pinruan, U. Lumyong, S., Hyde, K. D. \& Jones, E. B. G. (2007). Occurrence of fungi on tissues of the pe,atswamp palm Licuala longicalycata. Fungal Diversity, 25, 157-173.

Ramalho, C. I. (2008). Estrutura da Vegetação e distribuição espacial do licuri (Syagrus coronata (Mart.) Becc.) em dois municípios do Centro Norte da
Bahia, Brasil. Tese (Doutorado em Agronomia), Universidade Federal da Paraíba, Areia.

Santos, M. A. L., Vitória, N. S. \& Bezerra, J. L. (2016). Fungos Colonizando Palmeiras em Áreas de Caatinga do Sertão da Bahia. Agrotrópica, 28(1), 37-46. doi:10.21757/0103-3816.2016v28n1p37-46

Santos, M. A. L, Bezerra, J. L. \& Vitória, N. S. (2019a). Phaeoseptum aquaticum (Halotthiaceae), new record for American continent in a new host for science. Rodriguésia 70, e00282018. doi:10.1590/21757860201970080

Santos, M. A. L., Fortes, N. G. S., Silva, T. E. F. \& Vitória, N. S. (2019b). Ascomycota (lichenized and non-lichenized) on Syagrus coronata in the Caatinga biome: new and interesting records for Brazil and South America. Mycotaxon, 134, pp. 737. doi:10.5248/134.737

Santos, E. C. S. \& Vitória, N. S. (2017). Espécies de Ascomycota em Syagrus coronata (Mart.) Becc., Água Branca, Alagoas, Brasil. Revista Ouricuri, 7(1), 80-97.

Schultes, N. P., Murtishi, B. \& Li, D. W. (2017). Phylogenetic relationships of Chlamydomyces, Harzia, Olpitrichum and their sexual allies, Melanospora and Sphaerodes. Fungal Biology, 121(10), 890-904. doi:10.1016/j.funbio.2017.07.004

Sierra, D. (2006). Contribucion al estudio de los ascomicetes bitunicados de Cataluna. Acta Botanica Barcinonensia, 50, 5-434.

Sena, L. M. M. (2011). Conserve a Caatinga - O Bioma Caatinga. Vol. 1. Fortaleza: Associação Caatinga.

Silva, V. A., Andrade, L. H. C. \& Albuquerque, U. P. (2006). Revising the Cultural Significance index: The Case of the Fulni-ô in Northeastern Brazil. Field Methods 18, 98-108. doi:10.1177/1525822X05278025

Species Fungorum. (2019). Authors of Fungal Names.

$\begin{array}{llll}\text { Species } & \text { Link. } & \text { (2019). } & \text { Recuperado de }\end{array}$ http://www.splink.org.br/index?lang=pt

Tanaka, K., Hirayama, K., Yonezawa, H., Hatakeyama, S., Harada, Y., Sano, T.,...\& Hosoya, T. (2009). Molecular taxonomy of bambusicolous fungi: Tetraplosphaeriaceae, a new pleosporalean family with Tetraploa-like anamorphs. Studies in Mycology, 64, 175209. doi:10.3114/sim.2009.64.10

Taylor, J. E. \& Hyde, K. D. (2003). Microfungi of Tropical and Temperate Palms. Fungal Diversity Research Series 12.

Velloso, A. L., Sampaio, E. V. S. B. \& Pareyn, F. G. C. (2002). Ecorregiões: Propostas para o Bioma Caatinga. Recife, Associação Plantas do Nordeste-Nature Conservancy do Brasil. 2002.

Verma, R. K. (1996). Some new records of fungi associated with salfi palm (Caryota urens). Indian Phytopathol. 49, 22-25.

Vitoria, N. S., Bezerra, J. L., Gramacho, K. P. \& Luz, E. D. M. N. (2008). Camaroetella torrendiella comb. nov. e C. acrocomiae: agents etiológicos das lixas do coqueiro. Tropical Plant Pathology, 33(4), 295301.

Vitoria, N. S., Bezerra, J. L. \& Gramacho, K. P. (2010). A simplified DNA extraction method for PCR analysis of Camarotella spp. Brazilian Archives of Biology and Technology, 53(2), 249-252. doi: 10.1590/S1516-89132010000200001

Vitoria, N. S., Cavalcanti, M. A. Q. \& Bezerra, J. L. (2016a). Species of Astrosphaeriella and Fissuroma from palms: new records for South America and Brazil. Nova Hedwigia Band, 102(1-2), 129-140. doi:10.1127/nova_hedwigia/2015/0293

Vitoria, N. S., Cavalcanti, M. A. Q., Hyde, K. D. \& Bezerra, J. L. (2011a). Arecomyces new to Brazil, including A. attaleae sp. nov. Cryptogamie Mycologie, 32(1), 103-108. doi:10.7872/crym.v32.iss1.2012.103

Vitoria, N. S., Cavalcanti, M. A. Q., Hyde, K. D. \& Bezerra, J. L. (2012). Brunneiapiospora brasiliensis sp. nov. (Clypeosphaeriaceae) on palms from Brazil. Nova Hedwigia, 94 (1-2), 245-250. doi:10.1127/00295035/2012/0094-0245

Vitoria, N. S., Cavalcanti, M. A. Q., Luz, E. D. M. N. \& Bezerra, J. L. (2011b). Endocalyx melanoxanthus var. melanoxanthus (Ascomycota), a new to Brazil and three new hosts. Mycotaxon, 117, 09-113. doi:10.5248/117.109

Vitoria, N. S., Cavalcanti, M. A. Q., Santos, C. D., Pereira, J. \& Bezerra, J. L. (2013). Neolinocarpon attaleae sp. nov. on Attalea funifera (Arecaceae) from Brazil. Mycotaxon, 123, 141-145. doi:10.5248/123.141

Vitoria, N. S., Santos, M. A. L. \& Bezerra, J. L. 2019. Contribuições para o conhecimento de fungos (Ascomycota) em Mauritia flexuosa L.f. e Acrocomia intumescens Drude, Brasil. Revista Brasileira de Geografia Física vol.12, n.04 1252-12. doi:10.26848/rbgf.v12.4.p1252-1258

Vitoria, N. S., Santos, M. A. L. \& Fortes, N. G. S. 2016b. Comunidade fúngica de Syagrus coronata (Mart.) Becc: Ascomycota anamórficos e teleomórficos. In: Andrade, M.J.G.; Nogueira, E.M.S., Santos, C.A.B. 
(org.). Ecologia e Biodiversidade do Semiárido Nordestino. Vol. I BOTÂNICA. Paulo Afonso: SABEH, Cap. 2 , p. 35-45.

Wang, Y., et al. 2015. Overview of Stachybotrys (Memnoniella) and current species status. Fungal Diversity, 71, 17-83. doi:10.1007/s13225-0140319-0

Wijayawardene, N. N., Hyde, K. D., Lumbsch, T., Liu, J. K., Maharachchikumbura, S. S. N., Ekanayaka, A. H., Tian, Q. \& Phookamsak, R. 2018. Outline of Ascomycota - 2017. Fungal Diversity 88, 167-263. doi:10.1007/s13225-018-0394-8

Wu, B., Hussain, M., Zhang, W., Stadler, M., Liu, X. \& Xiang, M. 2019. Current insights into fungal species diversity and perspective on naming the environmental DNA sequences of fungi. Mycology, 10, 127-140. doi:10.1080/21501203.2019.1614106

Zhao, G. Z., Liu, X. Z. \& Wu, W. P. 2007. Helicosporous hyphomycetes from China. Fungal Diversity, 26: 313-524.

License: Creative Commons CC BY 4.0

This article was published with open access for distribution under the terms of the Creative Commons Attribution License, which allows unrestricted use, distribution, and reproduction in any medium, provided the original work is properly cited. 